\title{
INFLUENCE DE L'INERTIE DE L'EAU SUR LA STABILITÉ D'UN GROUPE HYDROÉLECTRIQUE \\ ISUITE *
}

\author{
P. ALMERAS \\ Ancien étève de l'École Polytechnique \\ Ingenteur aux Aleliers Neyret-Beylier \& Piccard-Pictet
}

\section{II. - Régulateur avec asservissement temporaire :}

Temps de relaxation du dash-pot: Considérons le schéma de la figure $\mathrm{n}^{\circ} 9$. Le point $C$ est ramené à une position fixe par un ressort de constante $C$. Soient $S$ et $s$ les surfaces du piston du dash-pot et de la lumière. Nous supposerons l'écoulement laminaire à travers la lumière du dash-pot; ceci n'est pas forcément vrai mais peut l'être pour de petites oscillations, en tout cas cela nous donnera une idée de la manière dont agit le dash-pot. Alờs la perte de charge dans la lumière est proportionnelle à la vitesse de l'huile. Soit $C^{\prime}$ le coefficient de proportionnalité. Soit figure $9 h_{0}$ la cote de régime du point $C, h$ sa cote actuelle et posons : $h-h_{0}=\Delta h_{\mathrm{C}}$; la force de rappel du ressort est $\mathrm{C} \Delta h_{\mathrm{C}}$, et par suite la pression dans le dash-pot est :

$$
\frac{C \Delta h_{\mathrm{f}}}{\mathrm{S}}
$$

la vitesse de l'huile dans la lumière est alors:

$$
\frac{\mathrm{CC} \Delta h_{\mathrm{C}}}{\mathrm{S}}
$$

et celle du piston du dash-pot par rapport au cylindre est :

$$
\frac{C C^{\prime} s}{\mathrm{~S}^{2}} \Delta h_{\mathrm{C}}
$$

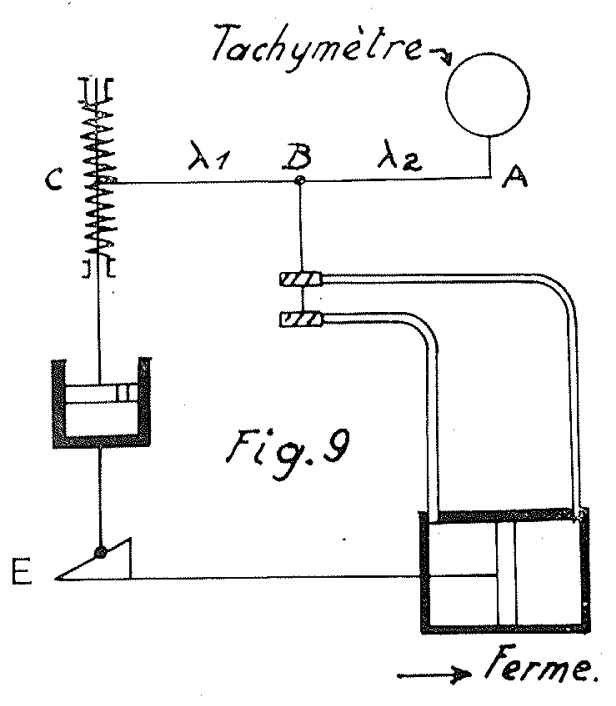

La quantité $\frac{\mathrm{CC}^{\prime} s}{\mathrm{~S}^{2}}$ a les dimensions de l'inverse d'un temps.

Nous poserons donc $T_{r}=\frac{\mathrm{S}^{2}}{\mathrm{CC}^{\prime} s}$ temps de relaxation du dash-pot.

* Voir notre numéro de Novembre-Janvier 1946. Des erreurs typographiques se sont glissées dans ce numéro. Nous nous en excusons auprès de nos lecteurs:
à la page 84 équation (1) lire $\left(y-y_{0}\right)$
aul licu de $\left(y+y_{0}\right)$
à la.page 87 3e ligne
lire $-\frac{\mathrm{L} \mathrm{V}_{\mu}}{g} \cdot \frac{d \mathrm{~V}}{d t \mathrm{~V}_{\mu}}$
au lieu $d e-\frac{\mathrm{L} \mathrm{V}_{p}}{\mathrm{~g}}-\frac{d}{d t} \frac{\mathrm{V}}{\mathrm{V}_{p}}$
à la page $89 \quad 2$ ligne
remplacer les 0
par des e
à la page $89 \quad 6$ ligne
lire $\left(\frac{y_{0} \Theta r}{2}+1\right) \times$ au lieu de $\left(\frac{y_{0} \Theta r}{2}+1\right)$ 
Interprétation physique du temps de relaxation: Soit un dash-pot à huile, dont le piston est ramené à une position déterminée par un ressort de constante $\mathrm{C}$. Supposons que nous écartons le piston de la quantité $h_{0}$ de sa position d'équilibre. Si nous négligeons les effets d'inertie de l'huile et du piston, nous trouvons facilement que le mouvement de retour du piston est régi par l'équation différentielle :

$$
\frac{d h}{h}=-\frac{d t}{\mathrm{~T}_{r}}
$$

$h$ étant au temps $t$ l'écart de la position du piston par rapport à sa position d'équilibre.

D'où :

$$
\frac{h}{h_{11}}=e^{-\frac{t}{\mathrm{~T}_{r}}}
$$

Le temps $T_{r}$ est donc le temps au bout duquel l'écart $h$ est égal à l'écart initial divisé par le nombre $e=2,72$.

On vérifie aussi que c'est le temps que mettrait le piston à rejoindre sa position d'équilibre s'il gardait pendant tout son mouvement sa vitesse initiale (proportionnelle à l'écart initial) :

$$
\frac{d h}{d t}=-\frac{h_{0}}{T_{r}^{d}}
$$

Le dash-pot sera d'autant plus serré et l'asservissement par conséquent plus rigide que le temps de relaxation sera plus grand. La mise en équation est maintenant facile.

Mise en équations :

On a vu que :

$$
\begin{aligned}
\frac{d y}{d t} & =-\mathrm{K}^{\prime} \Delta h_{\mathrm{B}} \\
& =-\mathrm{K}^{\prime} \frac{\lambda_{1}}{\lambda_{1}+\lambda_{\underline{2}}}\left(\frac{\Delta \omega_{1}}{\omega_{0}}+\frac{\lambda_{g}}{\lambda_{1} \mathrm{~K}} \Delta h_{\mathrm{C}}\right)
\end{aligned}
$$

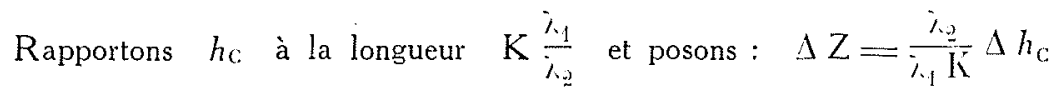

ceci devient :

$$
\frac{d y}{d t}=-\mathrm{K}_{0}\left(\frac{\Delta \omega}{\omega_{0}}+\Delta Z\right)=-\mathrm{K}_{0}(x-1+\Delta Z)
$$

Posons maintenant :

$$
\begin{array}{r}
x-1=\xi \\
y-y_{0}=\varepsilon \\
\Delta Z=\eta
\end{array}
$$

l'équation précédente devient :

$$
\frac{d \equiv}{d t}=-K_{0}\left(\xi+r_{i}\right)
$$

Les deux équations relatives au mouvement de la turbine et au coup de bélier sont les mêmes que précédemment :

$$
\begin{gathered}
\tau \frac{d \xi}{d t}=\Sigma+\frac{3}{2} y_{0} \zeta \\
\zeta=-\Theta\left(\frac{d \Xi}{d t}+\frac{1}{2} y_{0} \frac{d \zeta}{d t}\right)
\end{gathered}
$$

Ceci nous fait trois équations, il nous en faut une quatrième; nous l'aurons en écrivant que la vitesse du point $\mathrm{C}$ $\left(d h_{\mathrm{C}} / d t\right)$ est la somme de la vitesse du point $\mathrm{E}\left(m \frac{d y}{d t}\right)$ et de la vitesse du piston dans le cylindre du dashpot $\left(\frac{\mathrm{CC}^{\prime} s}{\mathrm{~S}} \Delta h_{\mathrm{C}}=\frac{1}{\mathrm{~T}_{r}} \Delta h_{\mathrm{C}}\right) \quad$ donc : $\quad \frac{d h_{\mathrm{C}}}{d l}=m \frac{d y}{d l}+\frac{1}{\mathrm{~T}_{r}} \Delta h_{\mathrm{C}}$ 
ou :

$$
\begin{gathered}
\frac{d z}{d t}=\frac{m}{\lambda_{1} K} \frac{d y}{d t}-\frac{1}{\mathrm{~T}_{r}} \Delta Z \\
\frac{d \eta_{r}}{d t}=\sigma \frac{d \xi}{d t}-\frac{1}{\mathrm{~T}_{r}} r_{r}
\end{gathered}
$$

ou :

Finalement le système qui régit le mouvement est le suivant :

$$
\begin{aligned}
& \frac{d \Sigma}{d l}=-K_{0}\left(\zeta+r_{i}\right) \\
& \tau \frac{d s}{d t}=z+\frac{3}{2} y_{11}= \\
& \because=-\theta\left(\frac{d \Sigma}{d t}+\frac{1}{2} y_{11} \frac{d \zeta}{d t}\right) \\
& \frac{d r_{1}}{d t}=\sigma \frac{d \equiv}{d t}-\frac{\mathrm{T}^{r}}{\mathrm{l}} r_{1}
\end{aligned}
$$

Nous le traitons comme précédemment, ce qui nous conduit à l'équation :

$$
\begin{aligned}
r^{4} \frac{y_{10} \Theta \tau}{2}+r^{3}=(1 & \left.+\frac{y_{10} \Theta \mathrm{K}_{10} \sigma}{2}+\frac{y_{01} \Theta}{2 \mathrm{~T}_{r}}\right)+r^{2}\left[\mathrm{~K}_{10}\left(=\sigma-y_{01} \Theta\right)+\frac{\tau}{\mathrm{T}_{r}}\right] \\
& +r \mathrm{~K}_{0}\left(1-\frac{y_{0} \Theta}{\mathrm{T}_{r}}\right)+\frac{\mathrm{K}_{01}}{\mathrm{~T}_{r}}=0
\end{aligned}
$$

de la forme:

$$
a_{1} r^{2}+a_{1} r^{3}+\alpha_{2} r^{2}+a_{3} r+\alpha_{4}=0
$$

Les conditions de stabilité s'écrivent :

qui se réduisent ici à :

$$
\begin{gathered}
\alpha_{0}>0 \quad \alpha_{1}>0 \quad \alpha_{2}>0 \quad \alpha_{3}>0 \quad \alpha_{4}>0 \\
\text { et } \alpha_{3}\left(\alpha_{1} \alpha_{2}-\alpha_{1} \alpha_{3}\right)-\alpha_{1}{ }^{2} \alpha_{1}>0
\end{gathered}
$$

(les autres coefficients étant automatiquement positifs)

$$
\text { et : } \quad \alpha_{3}\left(\alpha_{1} \alpha_{2}-\alpha_{10} \alpha_{3}\right)-\alpha_{1}^{2} \alpha_{1}>0
$$

Nous vérifierons tout à l'heure que les deux premières conditions sont entrainées par la dernière, qui se décompose ici en deux :

$$
\begin{gathered}
a_{23}>\frac{3+a_{3}}{2+a_{3}+\frac{1}{a_{1}}} \times \frac{2 a_{1}-2}{2 a_{1}-3-\frac{3}{a_{1} a_{3}}} \\
2 a_{1}>3+\frac{3}{a_{1} a_{3:}}
\end{gathered}
$$

où l’on a posé : $\quad a_{1}=\frac{T_{r}}{y_{11} \omega} \quad * \quad a_{2}=\frac{\tau \sigma}{y_{10} \Theta} \quad a_{31}=y_{10} \Theta \mathrm{K}_{10} \sigma$

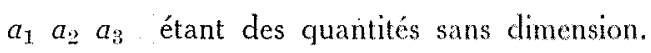

Portons $a_{1}$ en abscisses, $a_{2 .}$ en ordonnées, nous aurons des courbes d'équation :

$$
a_{2}=\frac{3+a_{3}}{2+a_{3}+\frac{1}{a_{1}}} \times \frac{2 a_{1}-2}{2 a_{1}-3-3-\frac{3}{a_{1} a_{3}}}
$$

et :

$$
2 a_{1}=3+\frac{3}{a_{1} a_{3}}
$$

courbes cotées en valeurs de $a_{3}$ (fig. 10). 
FIG. 10

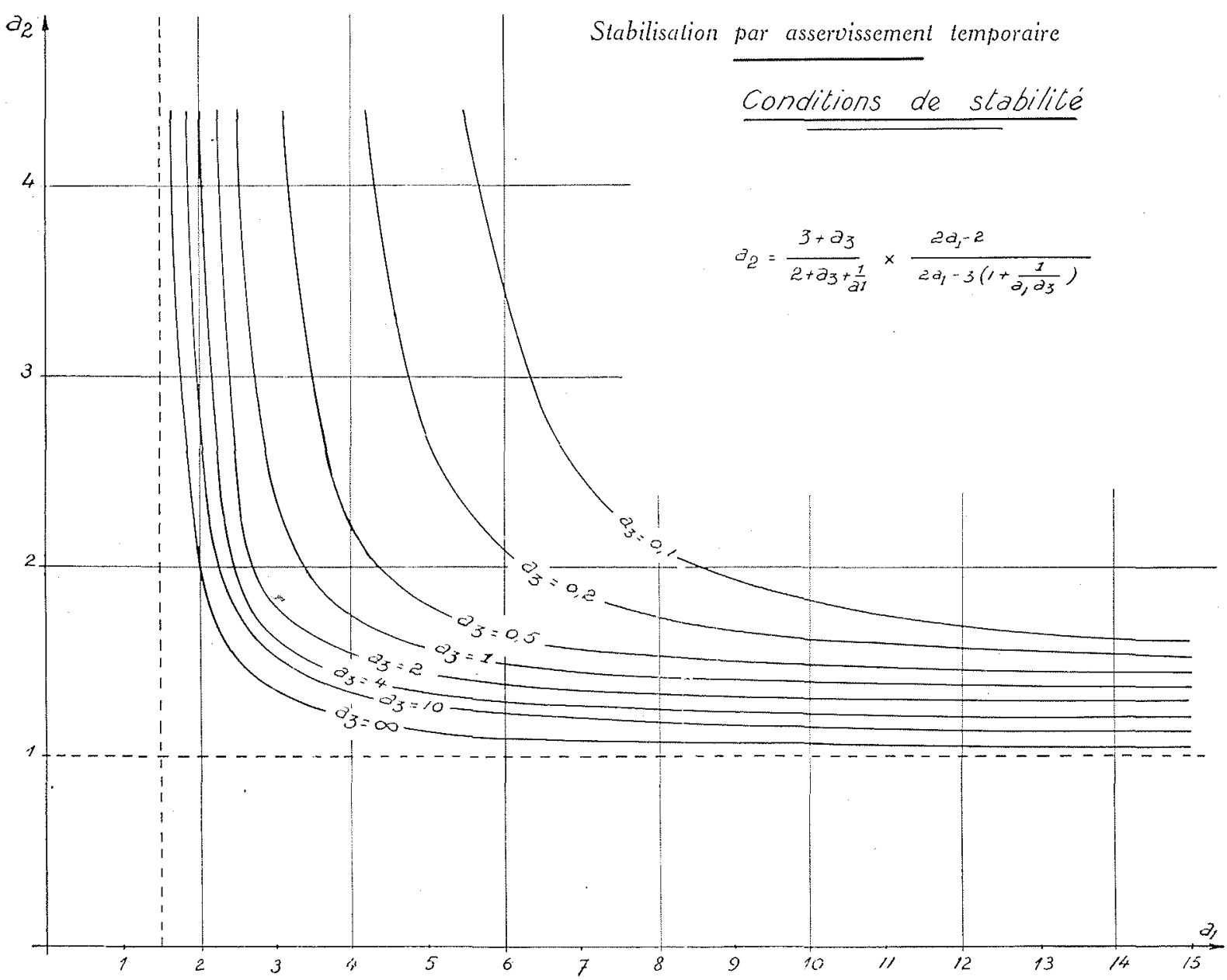

Les droites $(10$ bis $)$ sont les asymptotes verticales des courbes $(9$ bis $)$. Les conditions 9 et 10 signifient que le point figuratif $a_{1} a_{2}$ doit se trouver au-dessus de la courbe $(9$ bis $)$ cotée $a_{3}$, dont on ne considère que la portion à droite de son asymptote verticale. On vérifie d'ailleurs que, de ce fait, les conditions $\alpha_{2}>0$ et $\alpha_{s}>0$ se trouvent alors réalisées puisque $a_{1}$ et $a_{22}$ sont alors supérieurs à l'unité.

On voit que $a_{2}$ décroît de l'infini pour: $a_{1}=\frac{3 a_{3}+\sqrt{9 a_{3}{ }^{2}+24 a_{3}}}{4 a_{3}}$ (Tr faible) jusqu'à $\frac{3+a_{3}}{2+a_{3}}$ pour $a$, infini ( $T_{r}$ infini, asservissement rigide). On retrouve bien ici le résultat relatif à l'asservissement rigide.

On voit facilement que le criterium (9) est plus sévère à pleine charge $\left(y_{0}=1\right)$ qu'à vide; le criterium s'écrit, pour la pleine charge :

$$
\frac{\tau}{\Theta}>\frac{3+\Theta K_{0} \sigma}{2+\Theta K_{0} \sigma+\frac{\Theta}{T_{r}}} \times \frac{2 \frac{T_{r}}{\Theta}-2}{2 \frac{T_{r}}{\Theta}-3-\frac{3}{K_{0} T_{r} \sigma}}
$$

Lorsqu'on peut négliger l'effet du coup de bólier $(\Theta=0)$, le criterium (11) devient simplement $\sigma>0$.

Le graphique montre d'abord que le criterium est plus dur lorsque $K_{1}$ est faible ( $a_{3}$ faible) que lorsqu'il est grand ( $a_{3}$ grand); il $\mathrm{y}$ a donc intérêt a avoir une grande rapidité de réponse.

D'autre part, quand $a_{1}\left(\mathrm{c}\right.$ - -̀े-d. $\left.T_{n^{\prime}}\right)$ augmente, on voit que les courbes deviennent très rapidement horizontales et tendent vers leurs asymptotes.

L'équation (8) a 4 racines ( 2 réelles et 2 imaginaires conjuguées pour les valeurs habituellez des coefficients). Soient $r_{1}, r_{2}, r_{3} \pm i r_{1}$ ces racines. 
Si nous appelons $p$ le paramètre variable (qui peut être $\xi, \varepsilon, \zeta$, ou $\eta$ ), le mouvement est défini par :

$$
p=A e^{r_{1} t}+\mathrm{Be}^{r_{2} t}+\mathrm{Ce}^{r_{3} t} \operatorname{Cos}\left(r_{1} t+?\right)
$$

les coefficients A BC et $\varphi$ étant déterminés par les conditions initiales. Le mouvement comprend des termes apériodiques et un terme oscillatoire.

Considérons une installation donnée, où $\tau$ et $\Theta$ sont fixés; on augmentera la marge de stabilité demandée par les conditions (9) et (10) en augmentant : le degré d'asservissement $\sigma$, la rapidité de réponse $K_{0}$, le temps de relaxation $T_{r}$. Mais on voit immédiatement que, si l'augmentation de $\sigma$ et de $K_{11}$ entraîne bien un plus grand amortissement de tous les termes de la solution, car tous les coefficients de l'équation caractéristique se trouvent augmentés du même coup, par contre l'augmentation de $T_{r}$ (par diminution de l'eftort de rappel du ressort, ou étranglement de la lumière du dash-pot) entraîne notamment la diminution du terme constant $\alpha_{t}$ de l'équation (8), de sorte qu'une des conditions d'Hurwitz $\left(\alpha_{4}>0\right)$ se trouve de moins en moins réalisée.

De fait, en augmentant $T_{r}$, on augmente bien l'amortissement des oscillations, mais par contre on diminue une des racines réelles de l'équation caractéristique (quand $T$ tend vers $I^{\prime} \infty, \alpha_{t}$ tend vers zéro, et cette racine tend vers zéro), ce qui entraîne une diminution de l'amortissement d'un terme apériodique. On peut exprimer physiquement ceci en disant qu'on augmente alors le temps que met le groupe à reprendre sa vitesse de régime, après avoir visé provisoirement, pour amortir les oscillations, des vitesses différentes.

On voit d'ailleurs que, tout ennaugmentant outre mesure le temps de relaxation du dash-pot, on ne gagne pas grand'chose même en ce qui concerne la stabilisation des oscillations, car on s'éloigne peu de la courbe d'oscillations entretenues, laquelle est presque horizontale.

A la limite pour un régulateur infiniment rapide $\left(a_{3}=\mathrm{K}_{0}=\infty\right)$, la condition s'écrirait :

$$
\frac{\sigma \theta}{\Theta}=\frac{1-\frac{\Theta}{T_{r}}}{1-\frac{3}{2} \frac{\Theta}{T_{r}}}
$$

L'abaque de la figure 10 indique quelle valeur il y a lieu de choisir pour $\mathrm{T}_{r}$ avec les deux considérations opposées suivantes :

a) il faut le choisir le plus faible possible;

b) il faut le choisir suffisamment grand pour que l'effet de l'asservissement reste efficace. Le temps de relaxation minimum nécessaire pour qu'il en soit ainsi est d'autant plus faible que le régulateur a une réponse plus rapide.

\section{III. - RÉGulateur accéléro-tachymétrique :}

Le mouvement est régi par le système II :

$$
\begin{aligned}
& \frac{d \Xi}{d t}=-K_{0} \vdots-K_{1} \frac{d \Xi}{d t} \\
& -\frac{d \xi}{d l}=\varepsilon+\frac{3}{2} y_{i 1}= \\
& \zeta=-\Theta\left(\frac{d \varepsilon}{d l}+\frac{1}{2} y_{0} \frac{d \zeta}{d l}\right)
\end{aligned}
$$

qui traité comme le système I donne l'équation caractéristique :

ou :

$$
=r^{2}\left(1+\frac{y_{0} \Theta r}{2}\right)+\left(K_{0}+K_{1} r\right)\left(1-y_{11}(-r)=0\right.
$$

$$
r^{3}+r^{2} \frac{z-K_{1} y_{0} \Theta}{\frac{y_{0} \Theta z}{2}}+r \frac{K_{1}-K_{11} y_{11} \Theta}{\frac{y_{0} \Theta-}{2}}+\frac{K_{0}}{\frac{y_{0} \Theta-}{2}}=0
$$

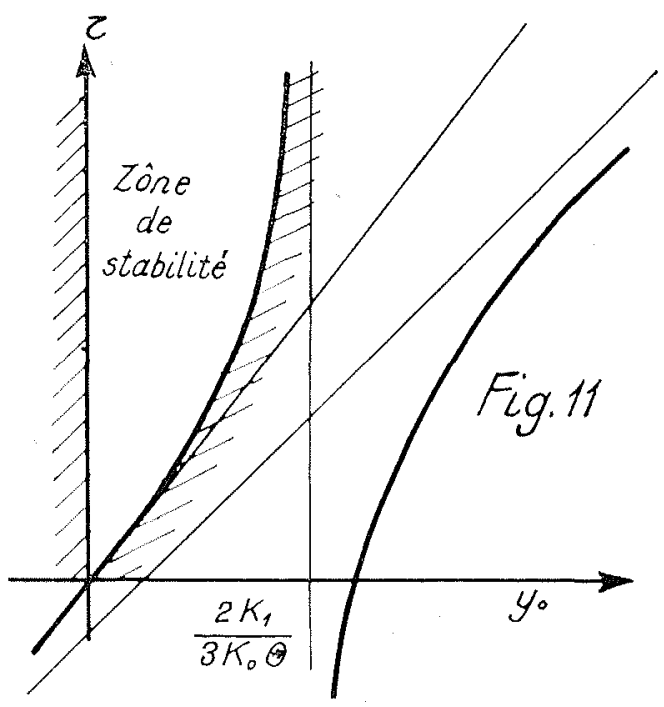


Les conditions de stabilité (HuRwitz) s'écrivent :

$$
\left.\begin{array}{l}
K_{0}>0 \\
\tau-K_{1} y_{0} \Theta>0 . \\
\tau\left(K_{1}-\frac{3}{2} K_{11} y_{10}(-)\right)-K_{1} y_{10}\left(-\left(K_{1}-K_{11} y_{01}(-)\right)>0\right.
\end{array}\right\}
$$

Portons $\tau$ en ordonnée et $y_{0}$ en abscisse (fig. 11).

Traçons la droite : $\tau=\mathrm{K}_{1} y_{0} \Theta$

et la courbe : $\tau=K_{1} y_{11} \Theta \frac{K_{1}-K_{11} y_{11} \Theta}{K_{1}-\frac{3}{2} K_{11} y_{11} \Theta}$

Pour que les deux inégalités (13) soient réalisées, il faut que le point figuratif se trouve dans la région non hachurée de la figure. Nous voyons sur cette figure que :
a) Si $\frac{2 K_{1}}{3 K_{11}}-1$
il y aura instabilité quel que soit $\tau$ pour toutes les valeurs de $y_{11}$ supérieures à $\frac{2 K_{1}}{3 K_{11}(-)}$
b) Si $\frac{2 K_{1}}{3 K_{01} \theta}>1$
il y aura stabilité pour toutes les valeurs de $y_{01}\left(0<y_{0}<1\right)$
si l'inégalité $\quad:>K_{1} \Theta \frac{K_{1}-K_{0} \Theta}{K_{1}-\frac{3}{2} K_{0} \Theta}$

est satisfaite.

Nous voyons sur la figure que les conditions de stabilité s'écrivent, pour l'ouverture $y_{0}$ :

$$
\frac{2 K_{1}}{3 K_{01} y_{01}(-)}>1 \quad \text { et }: \quad=>K_{1} y_{0} \Theta \frac{K_{1}-K_{11} y_{10} \Theta}{\left.K_{1}-\frac{3}{2} K_{11} y_{0}-1\right)}
$$

Pour qu'il y ait stabilité quelle que soit l'ouverture, il faut donc que:

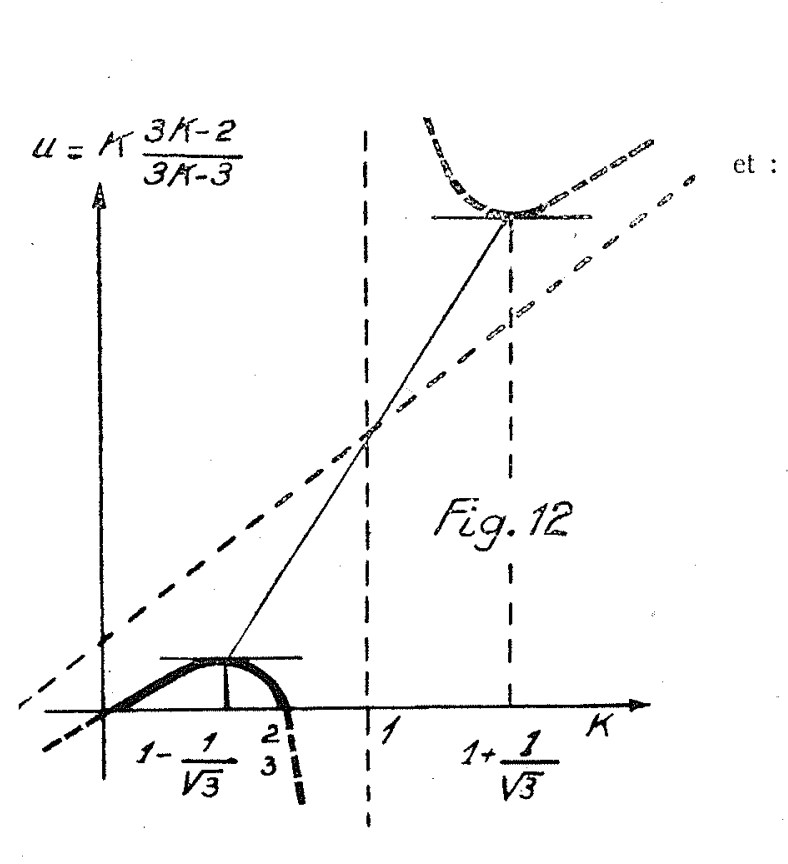

$$
\begin{gathered}
\frac{2 K_{1}}{3 K_{11} \Theta}>1 \\
\tau>K_{1} \Theta \frac{K_{1}-K_{11} \Theta}{K_{1}-\frac{3}{2} K_{0} \Theta}
\end{gathered}
$$

Cette inégalité peut s'écrire:

$$
K_{10} \Theta<K_{1} \frac{K_{1}-\frac{\tau}{\Theta}}{K_{1}-\frac{3}{2} \frac{\tau}{\Theta}}
$$

Posons $\mathrm{K}_{1}=\frac{3}{2} \frac{\overline{\bar{\theta}} \mathrm{G}}{\mathrm{K}} \mathrm{K}$, elle devient :

$$
K_{i} \Theta<\frac{3}{2} \div \mathrm{G} \frac{3 \mathrm{~K}-2}{3 \mathrm{~K}-3}
$$

Portons (fig. 12) en abscisse $\mathrm{K}$ et en ordonnée

$$
u=\mathrm{K} \frac{3 \mathrm{~K}-2}{3 \mathrm{~K}-3}
$$


Nous ne devons conserver de cette courbe que la portion pour laquelle $K$ est inférieur à $\frac{2}{3}$, puisque nous avons vu qu'une desconditions de stabilité est :

$$
\tau-\mathrm{K}_{1} \Theta>0 \quad \text { ou } \quad \mathrm{K}<\frac{2}{3}
$$

Cette courbe présente un maximum pour : $\quad K=1-\frac{\sqrt{3}}{3}$

et ce maximum a pour valeur : $\quad u=K \frac{3 K-2}{3 K-3}=\left(1-\frac{\sqrt{3}}{3}\right)^{2}$

Il est bien évident quiil y a intérêt, pour réaliser plus facilement la stabilité, à donner à $K_{1}$ la valeur correspondant à ce maximum :

$$
K_{1}=\frac{3}{2} \frac{i}{\theta} K=\frac{3}{2}\left(1-\frac{\sqrt{3}}{3}\right) \frac{\vdots}{\omega}
$$

Cette valeur, portée dans l'inégalité (14), donne finalement la condition de stabilité :

$$
\tau>3,74 \mathrm{~K}_{0} \Theta^{2}
$$

On peut vérifier que $K_{1}$ étant choisi comme indiqué, et l'inégalité (16) étant satisfaite, on a bien

$$
\frac{2 K_{1}}{3 K_{0}(-)}>1
$$

En résumé, une fois choisie la rapidité de réponse accślćrométrique optimum

$$
\mathrm{K}_{1}=\frac{3}{2}\left(1-\frac{\sqrt{3}}{3}\right)=
$$

- laquelle est d'autant plus faible que $\tau$ est plus petit et $\Theta$ plus grand -, on devra choisir une rapidité de réponse tachymétrique telle que :

$$
\mathrm{K}_{01}<0,267 \frac{\bar{\Sigma}}{\Theta_{2}}
$$

- donc, elle aussi d'autant plus petite que $\tau$ est plus petit et $\Theta$ plus grand -.

Mais il faut bien remarquer qu'on ne peut pas diminuer autant qu'on veut la rapidité de réponse $\mathrm{K}_{0}$, car, si cela permet de réaliser plus largement la condition de stabilité ci-clessus, cela diminue la marge de stabilité de la condition $\mathrm{K}_{0}>0$. De fait, on sait qu'un régulateur accélérométrique pur (sans tachymètre: $K_{0}=0$ ) n'est pas viable : en effet il ne règle nullement la fréquence. On se trouve ici en présence d'une circonstance analogue à celle du réglage avec asservissement temporaire : de même que pour stabiliser un groupe, on ne peut pas impunément augmenter la rigidité du dash-pot, de même en réglage accélérotachymérique on ne peut impunément diminuer la rapidité de réponse tachymétrique.

La condition (16) appelle les remarques suivantes: le temps de lancer (c'est-à-dire l'inertie du groupe) joue comme dans la régulation tachymétrique un rôle prépondérant, de même que $\Theta$. Par contre le rôle dévolu au degré d'asservissement est ici tenu par $\frac{1}{K_{11}}$, inverse de la rapidité de réponse du régulateur. Il y a ici intérêt à diminuer cette rapidité de réponse.

\section{CONCLUSIONS}

Les conditions de stabilité trouvées plus haut pour les groupes contrôlés par un régulateur tachymétrique avec asservissement temporaire ou par un régulateur accélérotachymétrique mettent en lumière les faits suivants :

Dans une installation déterminée, on augmentera la stabilité :

a) Dans les deux cas en augmentant l'inertie du groupe et en diminuant $\Theta$.

b) Dans le cas de l'asservissement temporaire : en augmentant le degré d'asservissement provisoire $\sigma$, et la rapidité de réponse $\mathrm{K}_{0}$. Par contre on ne peut pas augmenter impunément le temps de relaxation du dash-pot au-delà d'une certaine valeur (d'autant plus grande que le régulateur est plus rapide), car, si cela amortit effectivement les 
oscillations du groupe, cela diminue l'amortissement d'un terme apériodique — ce qui fait apparaître les défauts de l'asservissement permanent : retour de plus en plus lent à la fréquence de régime -

c) Dans le cas du système à accéléromètre : une fois le dosage accélérométrique optimum réalisé, en diminuant la rapidité de réponse $K_{0}$ du régulateur. Toutefois on ne peut pas diminuer outre mesure cette rapidité de réponse, - ce qui fait apparaître les défauts du réglage accélérométrique pur: incapacité à régler la fréquence, retour de plus en plus lent à la fréquence de régime -.

On voit que dans les deux cas la stabilité du réglage est obtenue au détriment de sa finesse : aussi bien la diminution de $K_{0}$ que l'augmentation du degré d'asservissement augmente l'écart maximum de fréquence consécutif à une variation donnée de la puissance demandée par le réseau.

On remarquera, à ce propos, que les partisans du système accélérotachymétrique ont coutume, pour le justifier, de se servir des deux arguments suivants :

a) Les constructeurs de régulateurs à asservissement temporaire font du réglage accélérométrique « sans le savoir 》. Mais dans le réglage tachymétrique on ne peut faire indéfiniment appel, pour augmenter la stabilité, à l'augmentation du degré d'asservissement, et à sa rigidité. Au contraire, en réglage accélérotachymétrique, on peut faire appel sans limitation à l'effet accélérométrique.

Or nous avons vu qu'il existe une valeur optimum de la rapidité de réponse accélérométrique $\mathrm{K}_{1}=\frac{3}{2}\left(1-\frac{\sqrt{3}}{3}\right) \frac{\tau}{\Theta}$ valeur à laquelle il y a intérêt à se tenir, et que d'ailleurs il ne saurait en aucun cas y avoir stabilité pour : $\quad K_{1}>\frac{3}{2} \frac{\bar{\sigma}}{G}$

D'autre part, si l'augmentation du degré d'asservissement augmente les écarts de fréquence, et si l'augmentation de sa rigidité $T_{r}$ augmente le temps que met le régulateur à retrouver apériodiquement la fréquence de régime, de même, en réglage accélérométrique, la diminution de $K_{0}$ présente à la fois ces deux inconvénients, — ce qui est peutêtre plus grave, puisqu'on ne peut ici jouer que sur un paramètre -

b) A rapidités de réponse égales un régulateur accélérométrique réagira beaucoup plus rapidement qu'un régulateur à asservissement, lors d'une variation de charge. En effet, dès le début, l'accélération a une valeur finie et son effet s'ajoute à l'effet tachymétrique, alors que l'asservissement s'oppose aux ordres donnés par le tachymètre et freine son action.

Notons que, dans l'esprit même de ceux qui l'énoncent, cette affrrmation ne vise que les cas où les variations de charge sont suffisamment faibles. En effet, dans les cas où elles sont notables, le tiroir de distribution se déplace suffisamment pour que la vitesse de vannage soit la vitesse maximum, et cette vitesse maximum eșt déterminée par des circonstances indépendantes du choix du régulateur (surpression maximum, survitesse maximum).

Bornons-nous donc aux faibles écarts de réglage. Nous avons vu que, en réglage accélérotachymétrique, les rapidités de réponse $K_{1}$ et $K_{0}$ sont toutes deux limitées par les conditions de stabilité :

$$
\begin{gathered}
K_{1}=\frac{3}{2}\left(1-\frac{1}{\sqrt{3}}\right) \frac{\sigma}{\theta} \\
K_{0}<0,267 \frac{\tau}{(-)^{2}}
\end{gathered}
$$

et ceci d'autant plus que $\Theta$ est plus grand.

Au contraire, en réglage tachymétrique, la condition de stabilité est d'autant plus facile à réaliser que la rapidité de réponse $\mathrm{K}_{0}$ est plus grande. Cette dernière n'est limitée que par des raisons de construction (encombrement du tiroir de distribution). On ne peut donc, en toute justice, comparer les deux régulateurs «à rapidités de réponse égales 》.

Toutefois pourrait-on dire, si limitée qu'elle soit par les considérations de stabilité, il n'en reste pas moins vrai que la vitesse de 《démarrage 》du vannage, en réglage accélérotachymétrique, n'est pas nulle, - l'accélération prenant une valeur finie au moment même de la variation de charge —, alors qu'elle est nulle en réglage tachymétrique l'écart de vitesse étant nul au début. On peut répondre à cela qu'il n'est pas du tout sûr que cela soit un avantage. On sait en effet que dans la plupart des installations (celles po: $r$ lesquelles le paramètre $\varrho$ d'Allırevr est supérieur à 0,5 , c'est-à-dire toutes les installations de basse et moyenne chute pour lesquelles nos calculs sont valables), une fermeture du vannage à vitesse finie commence par produire au début une augmentaticn de la puissance fournie par le groupe, donc un effet contraire à celui qu'elle « voudrait produire». Dès lors ce démarrage brutal du vannage ne 
fait qu'augmenter l'écart de fréquence qui suit une variation de charge donnée, puisque cette énergie superflue ainsi fournie par la turbine s'emmagasine sous forme d'énergie cinétique dans le groupe lui-même.

On reproche souvent aux régulateurs de turbines hydrauliques d'être incapables de régler. Il faut donc bien noter, à ce sujet, que cela ne vient pas de défauts dans la conception même de ces régulateurs (de quelque système qu'ils soient) mais de la nature même des choses : à savoir l'inertie du fluide moteur, l'eau. Nous aurons l'occasion de parler, plus loin, des conséquences qu'entraînent ces circonstances.

(A suive.)

\section{A propos de la théorie de M. Gaden}

Au moment oì notre précédent article passait sous presse, nous arons pris connaissance du livre de M. Gubx, intitulé "Contribution à l'étude des régulateurs automatiques de vitesse. Considérations sur le problème de la stabilité n.

Une lecture rapide de cet ouvrage, dont l'objet est le même que celui de notre étude, nous révéla des différences sensibles et des résulats apparemmen! clissemblables pour l'expression des conditions de stabilité.

Nous avions cru alors pouvoir attribuer ces différences à ce que M. Gankx aurait négligé, dans la mise en compte du coup de bélier, linteraction entre la surpression et le débit.

En fait, un examen plus approfondi, auquel nous avons pu nous livrer entre temps, nous a montré que la comparaison entre nos detux ćtules fait appel ì des considérations plus complexes que nous voudrions préciser ci-après:

Pour ce qui est du point signalé ci-clessus, relatif à l'interaction entre la pression et le débit, il y a lieu de souligner que cet elfet est parfaitement pris en compte dans les abarques que $M$. Gow obtient à partir de la théorie d'Arbiavi. Il n'y a done sur ce point aucun désaccord fondamental entre les deux théoties.

La méthode de calcul de M. Gows présente le grand avantage de pouvoir traiter d'une manière uniforme les installations de toules caractéristiques, quelles que soient la longueur de la contuite et la hauteur de chute.

Fn ce qui nous concerne, atu contraire, nous nous sonmes tout spécialement attaché au cals du coup de bélier en masse, $c 0$

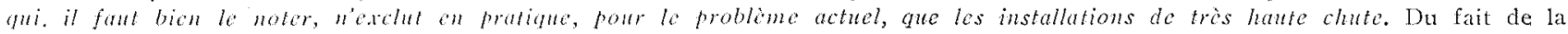
phus grande généralité des cas atuxquels doit s'applifuer sa méthode, M. Gabex est amené à introduire, dans ses conditions de stabilité, certains coefficients caractéristnt linfluence du coup de bélier, coeficients qui doivent être calculés par approximations staccessives a partir des ahaques auxiuels nous arons fat allusion plus haut,

En nous limitant au coup de bélér en masse, nous abontissons, pour notre part, à des résultats immédiatement explicites, permettant d'étudier d'une manière synthétique les divers atspects du phénomène.

En particulier. cette possibilité nous conduit à mettre en évidence l'existence d'une rapidité de réponse accélérométrique optimum; elle nous permet aussi de trater complètement le cas de lasservissment temporaire, gue M. Garmx a abordé en négligeant d'emblée un terme qui peut, dans la pratique, n’ètre pas toujours négligeable.

En défnitive, on voit, par le parallèle succinct gui précède, que les deux méthodes paraissent posséder chacune des avantages propres et des limitations. Un examen plus détaillé, qui sortirait du cadre de cet article, permet de montrer que leurs résultats concordent dans les domaines ou elles pewent s'appliquer simultanément.

P. A. 